\title{
Schatten $p$-norm based Principal Component Analysis
}

\author{
Heyou Chang ${ }^{1}$, Lei Luo ${ }^{1}$, Jian Yang ${ }^{1}$ and Meng Yang ${ }^{2}$ \\ ${ }^{1}$ School of Computer Science and Engineering, Nanjing University of Science and \\ Technology, Nanjing, P.R. China \\ ${ }^{2}$ Shenzhen University, Shenzhen,P.R. China
}

\begin{abstract}
Structured sparse PCA (SSPCA) is a new emerging method regularized by structured sparsity-inducing norms. However, these regularization terms are not necessarily optimal because of the noisy and irrelevant features embedded in predefined patterns. This paper presents a method called Schatten $p$-norm based principal component analysis $\left(\mathrm{S}_{p} \mathrm{PCA}\right)$ to learn interpretable and structured elements (or factors). In $\mathrm{S}_{p} \mathrm{PCA}$, a low-rank assumption is used to characterize structured elements in a two-dimensional matrix form. Compared to SSPCA, the low-rank assumption of $\mathrm{S}_{p} \mathrm{PCA}$ is more intuitive and effective for describing object parts of an image. Moreover, $\mathrm{S}_{p} \mathrm{PCA}$ can deal with some scenarios, where the dictionary element matrixes have complex structures. We also propose an efficient and simple optimization procedure to solve the problem. Extensive experiments on denoising of sparse structured signals and face recognition on different databases (e.g. AR, Extend Yale B and Multi-PIE) demonstrate the superior performance over some recently proposed methods.
\end{abstract}

Keywords: Structured sparse PCA, low rank, Schatten p-norm, ADMM

\section{Introduction}

Principal component analysis (PCA) [1] is a classical technique widely used in feature extraction and unsupervised dimensionality reduction. It aims to find an orthogonal transformation to convert a set of correlated variables into uncorrelated ones. In the last decade, several alternatives to PCA have been proposed, notably independent component analysis (ICA) [2] and non-negative matrix factorization (NMF) [3]. 
Since each principal component in PCA is a linear combination of all the original variables, it is often difficult to interpret the results. For solving this problem, Zou et al [4] proposed sparse PCA (SPCA) using the lasso (elastic net) to produce modified principal component. Christophe et al [5] proposed robust SPCA to make the analysis resistant to outlying observations. However, these methods seems inappropriate in many applications because they only constrain the size or the sparsity of the principal factors without considering the important structures. For example, the pixels of an image, the common most data in compute vision, are naturally organized on a grid. The structural information in the image is very helpful. Meanwhile, the supports of factors explaining the variability of images can be expected to be localized or connected, such as eyes or mouth in face images. These structured relationships among variables can help us better interpret data and provide new insights into the underlying processes. Some works has been reported in the context of regression and classification [6, 7], occlusion pattern learning [8], and back ground subtraction [9] by exploiting such structure. Particularly, Jenatton et al [10] proposed structured sparse PCA (SSPCA) to yield a structured and sparse formulation of principal component analysis by adding sparse and some priori structural constraints in elements. Nonetheless, the convex structured sparsity constraints in [10] may not be necessarily consist with real-world applications. In order to capture more flexible and general structure, Ren et al [11] introduced binary matrices as auxiliary variables and proposed Markov Random Field (MRF) based SSPCA ( $\mathrm{MS}^{2} \mathrm{PCA}$ ) for gene interaction. It is worth noting that the methods mentioned above worked off-line. Then Zoltan et al [12] proposed online group-structured dictionary learning (OSDL) to fit large or slowly varying systems. Although these methods could achieve structured local factors, the structures of factors need to be pre-given in advance, which is likely to be not adequate and accurate in many practical applications because of the noisy and irrelevant features.

Recently, low-rank hypothesis has become an effective technique in depicting the structure in data. For instance, Wright et al established a robust principal component analysis (RPCA) [13] method, which assumes the error matrix is sparse and the clean data matrix is low rank. As an important extension of RPCA, the low-rank representation (LRR) [14] was presented to recover the subspace structures among data samples. Given a data matrix, LRR seeks the lowest-rank representation among all the candidates that can represent the data samples as linear combinations of the bases in a given dictionary. Unlike RPCA and LRR, Zhang et al [15] focused on dealing 
with image data which were corrupted by continuous occlusion, and proposed double nuclear norm-based matrix decomposition (DNMD) to recover the low-rank data in image vector space and remove the low rank error in the image (matrix) space simultaneously. To make full use of the low-rank structural information of error image, Yang et al [16] proposed a two-dimensional image matrix based matrix regression model, named nuclear norm based matrix regression (NMR), to carry out the image representation and classification. To handle face images with mixed noise, i.e., the structural noise plus the sparse noise, Luo et al [17] proposed nuclear- $\mathrm{L}_{1}$ norm joint matrix regression $\left(\mathrm{NL}_{1} \mathrm{R}\right)$ model for face recognition with mixed noise, which are derived by using MAP (maximum a posteriori probability) estimation. All these methods use rank function to characterize the structural information in data. Additionally, to facilitate the computation, they replace the rank function with the nuclear norm. And the experimental results show that nuclear norm based models indeed obtain the effective low rank solutions in a variety of scenarios.

In this paper, we aim to learn the interpretable structural elements (e.g., mouth, eyes, or forehead in face images), which generally leads to a low-rank image in contrast to the full-rank original image. In [10 12, using the predefined structural constraints in factors is away from the actual situation and cannot depict complex structural information. Here we use nuclear norm to capture the structures in data. Good performance has been reported in 14[16] by using nuclear norm to depict low-rank structures. As we know, the nuclear norm of a matrix is equal to the $L_{1}$ norm of a vector formed by the singular values of the same matrix. Inspired by the experimental observations and theoretical guarantees showing superiority of $L_{p}$ quasi-norm minimization to $L_{1}$ minimization in compressive sampling (CS) [18], some approaches in [19, 20] replace the rank function with the Schatten- $p$ quasi-norm and verify that Schatten- $p$ quasi-norm minimization is superior to nuclear nor$\mathrm{m}$ minimization. Specially, Nie et al [21] proposed to jointly use Schatten $p$-norm and $L_{p}$-norm to approximate the rank minimization problem and enhance the robustness to outliers, and achieved promising performance on collaborative filtering and social network link prediction. This paper presents a Schatten $p$-norm based principal component analysis method to perfor$\mathrm{m}$ the image denoising and classification. Instead of pre-defining certain structures in the elements, the proposed model processes the elements in a two-dimensional matrix form and learns the structures effectively by using low-rank constraint. Compared with SSPCA, the pre-given structures are 
not required and more discriminative and complex structural variables can be captured. Alternating direction method of multipliers (ADMM) is utilized to solve the proposed model. We perform experiments on the denoising of sparse structured synthetic signals and face recognition on the AR [22], Extend Yale B [23] and MultiPIE [24] databases. The experimental results clearly demonstrate that the proposed method is more effective than stateof-the-art methods for image denoising and face recognition.

The rest of this paper is organized as follows. Section 2 presents the proposed $\mathrm{S}_{p} \mathrm{PCA}$ model and the optimization of $\mathrm{S}_{p} \mathrm{PCA}$. Section 3 conducts the experiments on denoising and faces recognition, and Section 4 concludes the paper.

Notations: The extended Schatten $p$-norm $(0<p<\infty)$ of a matrix $\mathbf{A} \in \mathcal{R}^{l \times m}$ is defined as $\|\mathbf{A}\|_{S_{p}}=\left(\sum_{i=1}^{\min \{l, m\}} \sigma_{i}^{p}\right)^{1 / p}=\left(\operatorname{tr}\left(\mathbf{A}^{T} \mathbf{A}\right)^{p / 2}\right)^{1 / p}$, where $\operatorname{tr}(\cdot)$ means the trace operator. The Schatten $p$-norm of matrix $\mathbf{A} \in \mathcal{R}^{l \times m}$ to the power $p$ is $\|\mathbf{A}\|_{S_{p}}^{p}=\sum_{i=1}^{\min \{l, m\}} \sigma_{i}^{p}=\operatorname{tr}\left(\mathbf{A}^{T} \mathbf{A}\right)^{p / 2}$. If $p=1$ and 2 , the Schatten $p$-norm becomes nuclear norm (denoted by $\|\cdot\|_{*}$ ) and Frobenius norm (denoted by $\left.\|\cdot\|_{F}\right)$, respectively. The extended $L_{p}$-norm $(0<p<\infty)$ of a vector $\mathbf{x} \in \mathcal{R}^{n \times 1}$ is denoted by $\|\mathbf{x}\|_{p}=\left(\sum_{i=1}^{n}\left|x_{i}\right|^{p}\right)^{1 / p}$, where $x_{i}$ is the $i$-th element of the vector $\mathbf{x}$.

Remark 1. When $p<1$, the extended Schatten $p$-norm is only a quasinorm. But for convenience, we still call it Schatten $p$-norm.

\section{The proposed model}

In this section, we first propose Schatten $p$-norm based principal component analysis ( $\mathrm{S}_{p} \mathrm{PCA}$, for short) model by using Schatten $p$-norm to constrain each element of the dictionary, and then apply ADMM to solve $\mathrm{S}_{p} \mathrm{PCA}$. Finally, we analyze the complexity and convergence of the proposed algorithm.

\subsection{Schatten p-norm based principal component analysis model}

Suppose that we are given a set of samples $\mathbf{X}=\left[\mathbf{x}_{1}, \mathbf{x}_{2}, \ldots, \mathbf{x}_{n}\right] \in \mathcal{R}^{t \times n}(t>$ $n)$, the dictionary learning problem is to find a dictionary $\mathbf{D}=\left[\mathbf{d}_{1}, \mathbf{d}_{2}, \ldots, \mathbf{d}_{K}\right] \in$ $\mathcal{R}^{t \times K}(t \gg K)$ and coefficient $\mathbf{Z}=\left[\mathbf{z}_{1}, \mathbf{z}_{2}, \ldots, \mathbf{z}_{n}\right] \in \mathcal{R}^{K \times n}$, such that $\mathbf{X} \approx$ DZ. The matrix product $\mathbf{D Z}$ is called a decomposition of $\mathbf{X}$. To address this problem, dictionary learning has been widely investigated during the last decade [25 28]. It is natural, when learning the dictionary, to penalize or constrain some norms or quasi-norms on $\mathbf{D}$ and $\mathbf{Z}$, respectively, to encode 
prior information about the decomposition of $\mathbf{X}$. Dictionary learning (also called matrix decomposition [29]) is a general problem that contains, e.g., PCA, ICA, NMF, among many others. In particular, Jenatton et al [10] casted SSPCA problem in the dictionary learning framework and considered some structural constraints to control the structure of the supports of dictionary elements. Zoltan et al [12] extended SSPCA and proposed online group-structured dictionary learning, which not only was online, but also could represent general overlapping group structures and deal with missing information at a time.

Although the above approaches integrate the idea of structured sparsity into modeling, which use the mixed $\left(L_{1}, L_{2}\right)$ norm to attempt to characterize the structure information of the overall dictionary $\mathbf{D}$, it actually destroys global structure of each element. As we know, for a face image which is spatially continuous, the global structure information is extremely significant for recognition task [15, 16], thus, it should not be ignored. To emphasize this information, we do not stretch each element into a vector form, but use low rank constraint to characterize each element and preserve its spatial structure, which induces a low rank principal component analysis model:

$$
\begin{array}{r}
\min _{\mathbf{D}, \mathbf{Z}}\|\mathbf{X}-\mathbf{D Z}\|_{F}^{2}+\lambda \sum_{i=1}^{K} \operatorname{rank}\left(\Psi\left(\mathbf{d}_{i}\right)\right) \\
\text { s.t. }\left\|\mathbf{Z}^{i}\right\|_{2} \leq 1, \forall i \in\{1,2, \cdots, K\}
\end{array}
$$

where $\lambda \geq 0$ is a regularization parameter, $\Psi(\mathbf{d})$ converts a vector $\mathbf{d} \in \mathcal{R}^{t}$ to a matrix in $\mathcal{R}^{l \times m}$ (suppose the input image size is $l \times m$ and $t=l \times m$ ), $\mathbf{Z}^{i}$ is the $i$-th row of $\mathbf{Z}$ to avoid trivial solutions ${ }^{1}$.

It is well-known that the rank minimization problem is difficult to solve since it is NP-hard. Recently, some scholars replace rank function using its convex envelope: nuclear norm [30]. However, the nuclear norm relaxation may deviate the solution away from the real solution of original rank minimization problem. As we know, when $p \rightarrow 0$, the Schatten $p$-norm of $\mathbf{X}$ becomes rank function of $\mathbf{X}$ under $0^{0}=0$, therefore, we choose to use Schatten $p$-norm $(p<1)$ to replace rank function to obtain a closer solution to

\footnotetext{
${ }^{1}$ For example, if using the nuclear norm or schatten $p$-norm to approximate rank function in Eq.(1), the objective can be decreased by respectively dividing and multiplying $\mathbf{d}_{i}$ and $\mathbf{Z}^{i}$ by a constant factor.
} 
problem (1). To this end, we consider the following problem:

$$
\begin{aligned}
\min _{\mathbf{D}, \mathbf{Z}}\|\mathbf{X}-\mathbf{D Z}\|_{F}^{2}+\lambda \sum_{i=1}^{K}\left\|\Psi\left(\mathbf{d}_{i}\right)\right\|_{S_{p}}^{p} \\
\quad \text { s.t. }\left\|\mathbf{Z}^{i}\right\|_{2} \leq 1, \forall i \in\{1,2, \cdots, K\}
\end{aligned}
$$

We turned to the setting where the parameter $p$ is in $(0,1)$. For the convenience of solving model (2), we chose $p=1 / 2$ here. Compared with nuclear norm, Schatten 1/2-norm is closer to rank function.

\subsection{Optimization of $S_{p} P C A$ model}

For the splitting, problem (2) can be converted to the following equivalent form:

$$
\begin{aligned}
& \min _{\mathbf{D}, \mathbf{Z}}\|\mathbf{X}-\mathbf{D Z}\|_{F}^{2}+\lambda \sum_{i=1}^{K}\left\|\Psi\left(\mathbf{v}_{i}\right)\right\|_{S_{p}}^{p} \\
& \quad \text { s.t. } \mathbf{D}=\mathbf{V},\left\|\mathbf{Z}^{i}\right\|_{2} \leq 1, \forall i \in\{1,2, \cdots, K\}
\end{aligned}
$$

The ALM method [21] is used to solve problem 3, which is equivalent to minimizing its augmented Lagrange function:

$$
\begin{aligned}
& \mathbf{J}=\|\mathbf{X}-\mathbf{D Z}\|_{F}^{2}+\lambda \sum_{i=1}^{K}\left\|\Psi\left(\mathbf{v}_{i}\right)\right\|_{S_{p}}^{p}+\operatorname{tr}\left(\mathbf{Y}^{T}(\mathbf{D}-\mathbf{V})\right)+\frac{\mu}{2}\|\mathbf{D}-\mathbf{V}\|_{F}^{2} \\
& \text { s.t. }\left\|\mathbf{Z}^{i}\right\|_{2} \leq 1, \forall i \in\{1,2, \cdots, K\}
\end{aligned}
$$

where $\mathbf{Y}$ is the Lagrange multiplier, $\mu>0$ is a penalty parameter. According to the idea of ADMM [31], we proceed by alternately fixing one variable and solving for the other, and iterating. In the following, we will state the detailed updating process.

Update of $\mathbf{Z}$. Due to the constraint of $\left\|\mathbf{Z}^{i}\right\|_{2} \leq 1$, we update $\mathbf{Z}$ row by row. For the updating of $\mathbf{Z}^{i}$, we assume that $\mathbf{D}$ and $\mathbf{Z}^{j}, j \neq i$ are fixed. Following the algorithms suggested by [32], $\mathbf{Z}$ can be updated by

$$
\begin{aligned}
\hat{\mathbf{Z}} & =\underset{\mathbf{Z}}{\arg \min }\|\mathbf{X}-\mathbf{D Z}\|_{F}^{2} \quad \text { s.t. }\left\|\mathbf{Z}^{i}\right\|_{2} \leq 1, \forall i \in\{1,2, \cdots, K\} \\
& =\underset{\left\|\mathbf{Z}^{i}\right\|_{2} \leq 1}{\arg \min }\left\|\mathbf{Z}^{i}-\right\| \mathbf{d}_{i}\left\|_{2}^{-2}\left(\mathbf{d}_{i}^{T} \mathbf{X}-\mathbf{d}_{i}^{T} \sum_{j \neq i} \mathbf{d}_{j} \mathbf{Z}^{j}\right)\right\|_{2}^{2} \\
& =\underset{\left\|\mathbf{Z}^{i}\right\|_{2} \leq 1}{\arg \min }\left\|\mathbf{Z}^{i}-\mathbf{w}\right\|_{2}^{2}
\end{aligned}
$$


where $\mathbf{w}=\left\|\mathbf{d}_{i}\right\|_{2}^{-2}\left(\mathbf{d}_{i}^{T} \mathbf{X}-\mathbf{d}_{i}^{T} \sum_{j \neq i} \mathbf{d}_{j} \mathbf{Z}^{j}\right)$. Problem (5) is actually a standard projection problem, which can be solved simply by projecting $\mathbf{w}$ onto the unit ball of $l_{2}$ norm: $\left\{\hat{\mathbf{Z}}^{i}:\left\|\hat{\mathbf{Z}}^{i}\right\|_{2} \leq 1\right\}$.

Update of $\mathbf{D}$. Fixing $\mathbf{Z}$ and $\mathbf{V}, \mathbf{D}$ can be updated by

$$
\hat{\mathbf{D}}=\underset{\mathbf{D}}{\arg \min }\|\mathbf{X}-\mathbf{D} \mathbf{Z}\|_{F}^{2}+\operatorname{tr}\left(\mathbf{Y}^{T}(\mathbf{D}-\mathbf{V})\right)+\frac{\mu}{2}\|\mathbf{D}-\mathbf{V}\|_{F}^{2}
$$

Since the objective function in subproblem (6) is differentiable, the optimal solution of (6) can be obtained by taking its stationary point, that is,

$$
\hat{\mathbf{D}}=\left(\mathbf{X Z}^{\mathbf{T}}+\frac{\mu \mathbf{V}-\mathbf{Y}}{2}\right)\left(\mathbf{Z Z}^{T}+\frac{\mu}{2} \mathbf{I}\right)^{-1}
$$

Update of $\mathbf{V}$. In order to update $\mathbf{V}$, we need to introduce a single value function thresholding theorem based on Schatten 1/2 norm. Firstly, by [33], we have following Lemma:

Lemma 1. The $L_{1 / 2}$ regularization problem:

$$
\min _{\sigma \geq 0} \frac{1}{2}(\sigma-\alpha)^{2}+\eta \sigma^{1 / 2}
$$

has the following closed-form solution:

$$
\sigma^{*}= \begin{cases}\frac{2}{3}|\alpha|\left(1+\cos \left(\frac{2 \pi}{3}-\frac{2 \varphi(\alpha)}{3}\right)\right), & \text { if } \alpha>w(\eta) \\ 0, & \text { if } \alpha \leq w(\eta)\end{cases}
$$

where $\varphi(\alpha)=\arccos \left(\frac{\eta}{4}\left(\frac{|\alpha|}{3}\right)^{(-3 / 2)}\right), w(\eta)=\frac{\sqrt[3]{54}}{4}(2 \eta)^{2 / 3}$.

By the above Lemma, we can obtain the following Theorem 1.

Theorem 1. Let $\eta>0$ be a constant and $\mathbf{G} \in \mathcal{R}^{l \times m}$ be a given matrix with rank $r$, the optimal solution to

$$
\min _{\mathbf{A}}\left(\eta\|\mathbf{X}\|_{S_{1 / 2}}^{1 / 2}+\frac{1}{2}\|\mathbf{A}-\mathbf{G}\|_{F}^{2}\right)
$$

is $\mathbf{A}^{*}=\Gamma_{\eta}^{1 / 2}(\mathbf{G}):=\mathbf{U} \boldsymbol{\Omega}_{\eta}^{1 / 2}(\mathbf{G}) \mathbf{V}^{T}, \boldsymbol{\Omega}_{\eta}^{1 / 2}(\mathbf{G})=\operatorname{diag}\left(\varpi\left(\sigma_{i}\right) \cdot \varepsilon\right)$. where $\Gamma_{\eta}^{1 / 2}(\cdot)$ is a single value function thresholding operator based on Schatten 1/2-norm, $\sigma_{1}, \sigma_{2}, \ldots, \sigma_{r}$ are positive singular values of $G, \boldsymbol{\Omega}=\operatorname{diag}\left(\left\{\sigma_{i}\right\}_{1 \leq i \leq r}\right)$, $\mathbf{U}_{l \times r}$ and $\mathbf{V}_{m \times r}$ are the corresponding matrices with orthogonal columns. $\varpi\left(\sigma_{i}\right)=\frac{2}{3}\left|\sigma_{i}\right|\left(1+\cos \left(\frac{2 \pi}{3}-\frac{2 \varphi\left(\sigma_{i}\right)}{3}\right)\right) \mid, \varphi\left(\sigma_{i}\right)=\arccos \left(\frac{\eta}{4}\left(\frac{\sigma_{i}}{3}\right)^{-3 / 2}\right), \varepsilon=$ $\left\{\begin{array}{ll}1, & \sigma_{i}>w(\eta) \\ 0, & 0 \geq \sigma_{i} \leq w(\eta)\end{array}\right.$, and $w(\eta)=\frac{\sqrt[3]{56}}{4}(2 \eta)^{2 / 3}$. 
Proof. Let the optimal solution to (8) be $\mathbf{A}^{*}$, and let the SVD of $\mathbf{A}^{*}$ and $\mathbf{G}$ be $\mathbf{A}^{*}=\mathbf{U} \boldsymbol{\Sigma} \mathbf{V}^{T}$ and $\mathbf{G}=\mathbf{Q} \boldsymbol{\Delta} \mathbf{R}^{T}$, respectively. Denote the objective in the problem (8) by $f(\mathbf{A})$, then

$$
\begin{aligned}
f(\mathbf{A}) & =f(\mathbf{U}, \boldsymbol{\Sigma}, \mathbf{V}) \\
& =\eta\left\|\mathbf{U} \boldsymbol{\Sigma} \mathbf{V}^{T}\right\|_{S_{p}}^{p}+\frac{1}{2}\left\|\mathbf{U} \boldsymbol{\Sigma} \mathbf{V}^{T}-\mathbf{Q} \boldsymbol{\Delta} \mathbf{R}^{T}\right\|_{F}^{2} \\
& =\eta \operatorname{tr}\left(\boldsymbol{\Sigma}^{\mathbf{p}}\right)+\frac{1}{2}\left[\operatorname{tr}\left(\boldsymbol{\Sigma}^{T} \boldsymbol{\Sigma}\right)+\operatorname{tr}\left(\boldsymbol{\Delta}^{T} \boldsymbol{\Delta}\right)-2 \operatorname{tr}\left(\mathbf{A}^{T} \mathbf{G}\right)\right] \\
& \geq \eta \operatorname{tr}\left(\boldsymbol{\Sigma}^{\mathbf{p}}\right)+\frac{1}{2}\left[\operatorname{tr}\left(\boldsymbol{\Sigma}^{T} \boldsymbol{\Sigma}\right)+\operatorname{tr}\left(\boldsymbol{\Delta}^{T} \boldsymbol{\Delta}\right)-2 \operatorname{tr}\left(\boldsymbol{\Sigma}^{T} \boldsymbol{\Delta}\right)\right] \\
& =\eta \operatorname{tr}\left(\boldsymbol{\Sigma}^{\mathbf{p}}\right)+\frac{1}{2}\|\boldsymbol{\Sigma}-\boldsymbol{\Delta}\|_{F}^{2} \\
& =\sum_{i}\left(\eta \sigma_{i}^{p}+\frac{1}{2}\left(\sigma_{i}-\alpha_{i}\right)^{2}\right)
\end{aligned}
$$

Thus the problem (8) can get its minimization when $\mathbf{U}=\mathbf{Q}$ and $\mathbf{V}=\mathbf{R}$. In addition, minimizing $f(\mathbf{A})$ is reduced to minimizing the following problem:

$$
\min _{\sigma \geq 0} \sum_{i} \eta \sigma_{i}^{p}+\frac{1}{2}\left(\sigma_{i}-\alpha_{i}\right)^{2}
$$

By Lemma 1, 10 has the following closed-form solution:

$$
\sigma_{i}^{*}= \begin{cases}\frac{2}{3}\left|\alpha_{i}\right|\left(1+\cos \left(\frac{2 \pi}{3}-\frac{2 \varphi\left(\alpha_{i}\right)}{3}\right)\right), & \text { if } \alpha_{i}>w(\eta) \\ 0, & \text { if } \alpha_{i} \leq w(\eta)\end{cases}
$$

Thus, we can obtain the optimal solution $\mathbf{A}^{*}$ to problem $(8)$ is $\mathbf{A}^{*}=\Gamma_{\eta}^{1 / 2}(\mathbf{G}):=$ $\mathbf{U} \Omega_{\eta}^{1 / 2}(\mathbf{G}) \mathbf{V}^{T}$.

The algorithm of the proposed $\mathrm{S}_{p} \mathrm{PCA}$ is summarized in Algorithm 1 .

\subsection{Time Complexity and Convergence}

Eq. (4) is not jointly convex in $\mathbf{Z}, \mathbf{D}$ and $\mathbf{V}$, but it is convex with either of them with the others fixed. In the proposed $\mathrm{S}_{p} \mathrm{PCA}$ algorithm, the time complexity of updating $\mathbf{Z}$ and $\mathbf{D}$ is both $O(\operatorname{tnK})$, where $t(t=l \times m)$ is the feature dimensionality, $n$ is the number of samples and $K$ is the dictionary size. The main running time of updating $\mathbf{V}$ is consumed in performing SVD on the small matrix of the size $l \times m$, and some matrix multiplications. The 


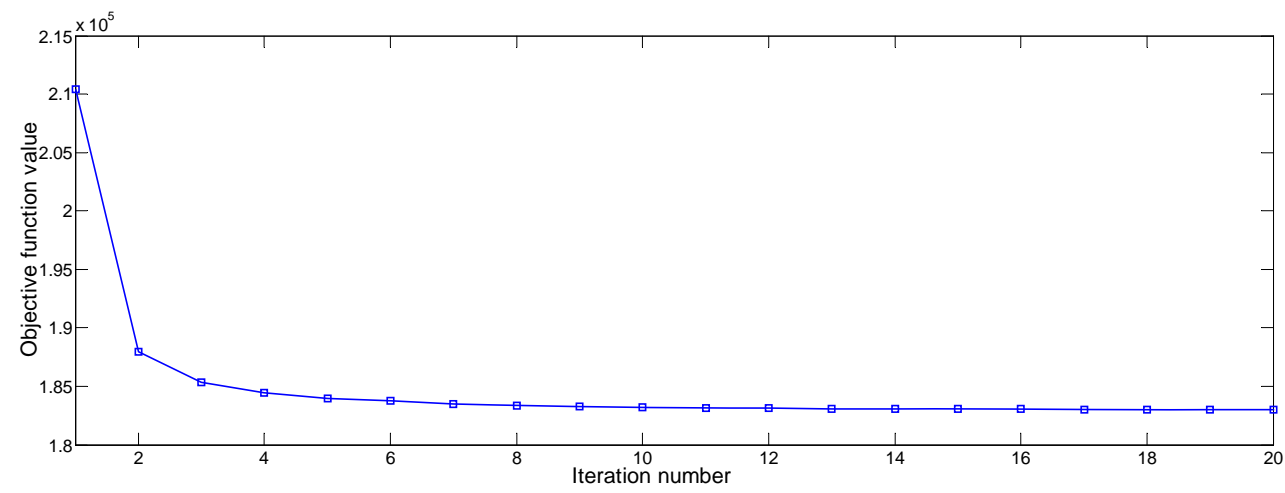

Figure 1: An example of $\mathrm{S}_{p} \mathrm{PCA}$ minimization process on denoising of synthetic signals

time complexity of performing SVD is $O\left(l m^{2}\right)$ (we assume that $m \leq l$ ). Thus, the total time complexity of the proposed algorithm is $O(t(n+m) K)$, where $t>n \gg\{K, m\}$. Since the update of $\mathbf{Z}$ and $\mathbf{D}$ are both convex, we can obtain easily the global optimal solutions for these two problems. The update of $\mathbf{V}$ has closed-solution, according to the analysis in [34, 35], we know that the proposed algorithm is convergent. In addition,the objective function value versus the iteration number on denoising of synthetic signals are shown as Fig.1.

\section{Experiments and results}

In this section, we verify the performance of our method on the denoising of synthetic signals and then the application to face recognition. Since $\mathrm{S}_{p} \mathrm{PCA}$ is an unsupervised method, to be fair, we compared $\mathrm{S}_{p} \mathrm{PCA}$ with some unsupervised dimensionality reduction methods, such as RPCA-OM [36], SSPCA [10, SPCA [4, NMF [3, ICA and PCA. In all experiments, the parameter $\lambda$ in learning phase is determined via cross-validation. After calculating $\mathbf{D}$ and $\mathbf{Z}$ in Eq.(2), the low-dimensional representation of a query sample $\mathbf{y}$ can be easily computed by $\hat{\mathbf{z}}=\left(\mathbf{D}^{T} \mathbf{D}+\zeta \mathbf{I}\right)^{-1} \mathbf{D}^{T} \mathbf{y}$, where $\zeta$ is a very small constant (e.g. $\left.1 e^{-4}\right)$. As [10], we applied k-nearest-neighbors classifier $(\mathrm{k}-\mathrm{NN})$ on the obtained $\hat{\mathbf{z}}$ and $\mathbf{Z}$ for face classification. 


\subsection{Denoising of Synthetic Signals}

We first generated the sparse and structured dictionary atoms $\mathbf{D}=$ $\left[\mathbf{d}_{1}, \mathbf{d}_{2}, \mathbf{d}_{3}\right] \in \mathcal{R}^{900 \times 3}$, which represent car, bottle and crow, respectively. Then the signals were generated by the following noisy linear model:

$$
z_{1} \mathbf{d}_{1}+z_{2} \mathbf{d}_{2}+z_{3} \mathbf{d}_{3}+\varepsilon \in \mathcal{R}^{900}
$$

The coefficients $\left[z_{1}, z_{2}, z_{3}\right]$ were generated according to a centered Gaussian distribution with the following covariance matrix $\Lambda$, where

$$
\Lambda=\left[\begin{array}{ccc}
1 & 0 & 0.5 \\
0 & 1 & 0.5 \\
0.5 & 0.5 & 1
\end{array}\right]
$$

The components of the noise vector $\varepsilon$ were independent and identically distributed according to a centered Gaussian distribution with its variance set to obtain a signal-to-noise ratio (SNR) of 0.5 .

Firstly, we learnt the dictionary with $K=3$ atoms from 500 of such signals, which seams a reasonable choice of $\mathrm{K}$ in an attempt to recover the underlying (in this case, known) structure of $\mathbf{D}$. Based on the learnt dictionary $\mathbf{D}$, we denoised 1000 new signals generated in the same way. It is difficult to learn the dictionary because of the high level noise and small number of signals (i.e. 500 signals against 900 variables). The reconstruction error was calculated by $\Upsilon=\frac{\|\mathbf{X}-\overline{\mathbf{X}}\|_{F}}{\sqrt{1000}}$, where $\mathbf{X}$ were the clear signals, and $\overline{\mathbf{X}}$ represented the reconstructed signals. The results of the denoising for PCA, SPCA, SSPCA, RPCA-OM and the proposed $\mathrm{S}_{p} \mathrm{PCA}$ are listed in Table 1 .

Table 1: Average and standard deviation of the normalized reconstruction error

\begin{tabular}{ccccc}
\hline PCA & SPCA & SSPCA & RPCA-OM & S $_{p}$ PCA \\
\hline $3.04 \pm 0.008$ & $4.00 \pm 0.001$ & $4.71 \pm 0.026$ & $2.99 \pm 0.004$ & $\mathbf{1 . 3 5} \pm \mathbf{0 . 0 0 1}$ \\
\hline
\end{tabular}

As shown on Fig,2, the dictionary elements learnt by PCA are very scattered and uninterpretable; SPCA learns three similar elements and cannot separate them; although SSPCA can learn three separated dictionary elements, the structure in each element is far away from the true structure. The proposed $\mathrm{S}_{p} \mathrm{PCA}$ can recover the structure of $\mathbf{D}$ mostly, which shows 


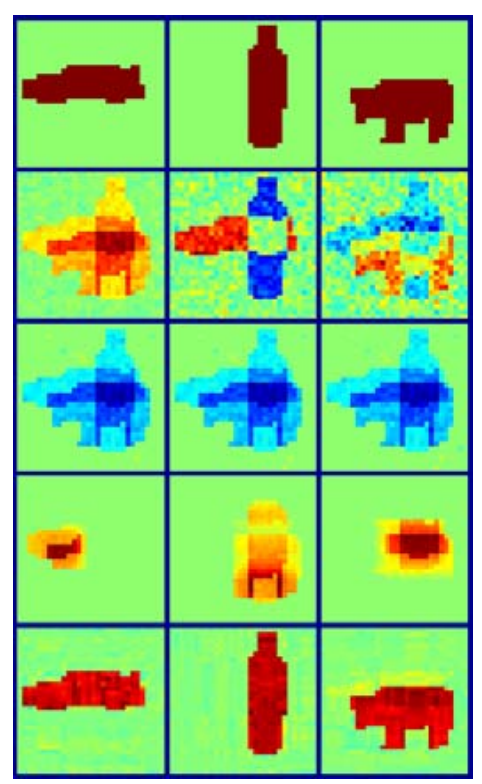

Figure 2: Top row: original dictionary $\mathbf{D} \in \mathcal{R}^{900 \times 3}$. From the second row to the bottom row: dictionary recovered by PCA,SPCA, SSPCA and $\mathrm{S}_{p} \mathrm{PCA}$

the effectiveness of the low rank constraint. Meanwhile, it is also noted that compared with SSPCA, the structure of each element learnt by $\mathrm{S}_{p} \mathrm{PCA}$ is closer to the true structure (i.e., the first row in Fig.2). Furthermore, the results in Table 1 demonstrate that the proposed $\mathrm{S}_{p} \mathrm{PCA}$ indeed performs better than other methods.

\subsection{Face classification}

To verify the effectiveness of our method to real face classification, experiments on three different face databases (i.e., AR, Extend YaleB and Multi-PIE) were done.

\subsubsection{AR database}

As [10], we chose a subset from AR database [22], which consists of 2600 face images, corresponding to 100 individuals (50 males and 50 females). For each subject, there are two sessions, including 14 non-occluded poses and 12 occluded ones (the occlusions are due to sunglasses and scarves). In our experiments, the face images were resized to $38 \times 27$. The non-occluded images were used to learn the dictionary $\mathbf{D}$ and the occluded faces were used 


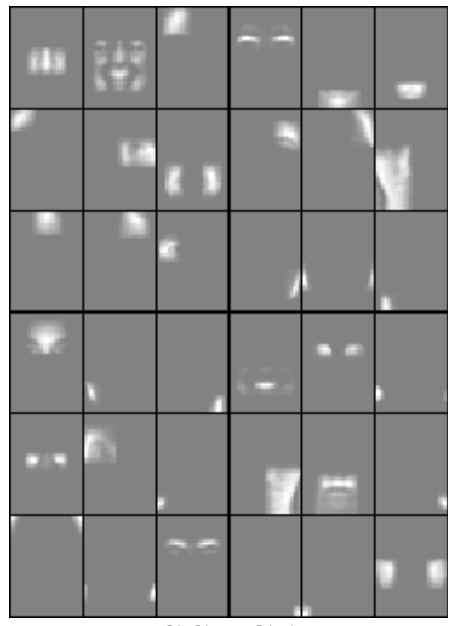

SSPCA

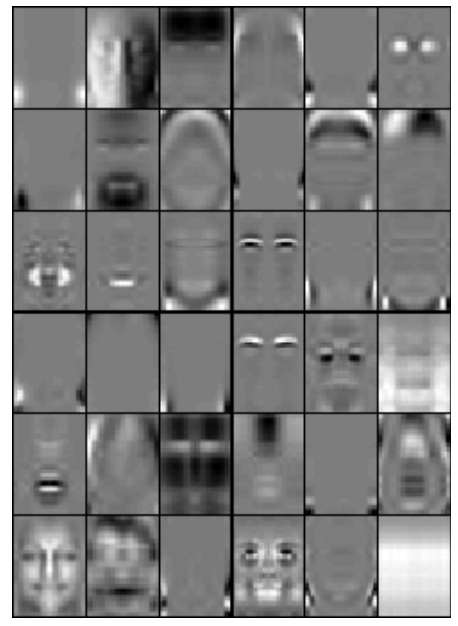

$\mathrm{S}_{p} \mathrm{PCA}$

Figure 3: The learnt dictionaries of faces with 36 elements on AR database

for testing. Fig. 3 shows examples of learnt dictionaries with 36 elements for SSPCA and $\mathrm{S}_{p} \mathrm{PCA}$. We can observe that the dictionary elements learnt by SSPCA correspond to local patches of faces, but some global structural information is missed. While the proposed $\mathrm{S}_{p} \mathrm{PCA}$ not only captured local structures of faces, such as mouth and eyes, but also some global structures, such as the whole contour of face.

We also quantitatively compared $\mathrm{S}_{p} \mathrm{PCA}$ with RPCA-OM, SSPCA, SPCA, NMF, ICA and PCA on face recognition problem. As [10, for different sizes $^{2}$ of the dictionary, we applied each of the aforementioned dimensionality reduction techniques to the no-occluded faces to learn the dictionary $\mathbf{D}$ and the corresponding coefficients $\mathbf{Z}$. Keeping the learnt dictionary $\mathbf{D}$, we decomposed the occluded faces on $\mathbf{D}$. Based on the obtained low-dimensional representations $\mathbf{Z}$, we classified the occluded faces utilizing the $\mathrm{kNN}$ classifier. In our experiments, the number of the neighbors was fixed as 1. From Fig 4 , we can see that the proposed method overall achieves the better performance than other methods except that it is slightly worse than SSPCA when the dictionary size is smaller than 20 . The best recognition rate of $\mathrm{S}_{p} \mathrm{PCA}$ is $71.42 \%$, compared with $36.83 \%$ for RPCA-OM, $57.08 \%$ for SSPCA, $20.08 \%$

\footnotetext{
${ }^{2}$ dictionary size $=\{10,20,30,36,40,50,60,70,80,90,100,110,120,130,140,150\}$
} 


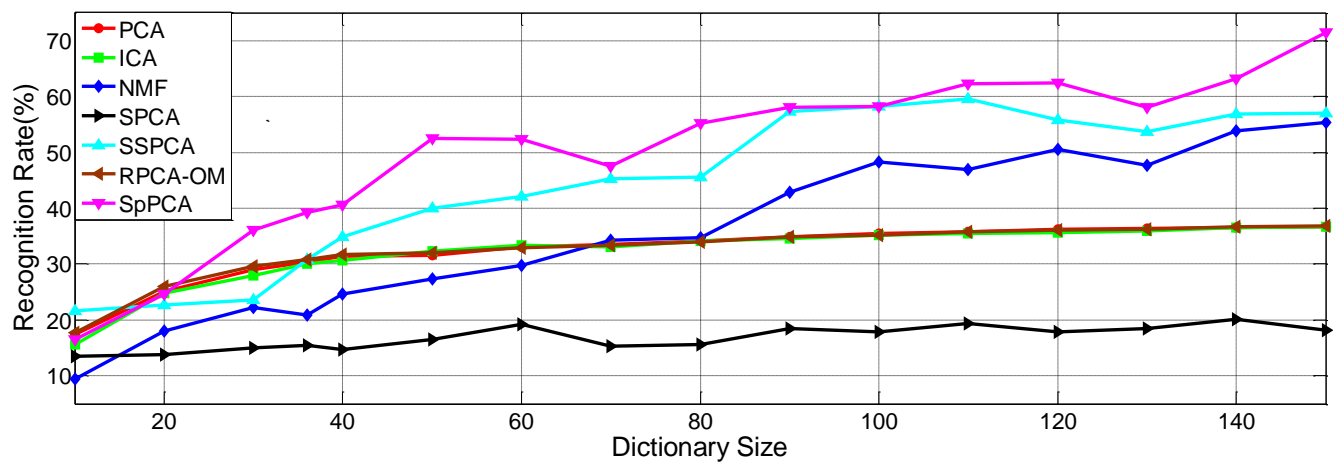

Figure 4: Classification accuracy versus dictionary size on AR database

for SPCA, $53.33 \%$ for NMF, $36.75 \%$ for ICA and $36.67 \%$ for PCA,

\subsubsection{Extended YaleB database}

The Extended YaleB database [23] contains about 2,414 frontal-face images of 38 individuals (around 59-64 images for each person). The cropped and normalized $192 \times 168$ face images were captured under various laboratorycontrolled lighting conditions. The face images was resized to $96 \times 84$. We randomly split the database into two halves. One half was used for training, and the other half for testing. The procedure is repeated 5 times and the mean accuracy is reported in Fig.5. It can be seen that the proposed $\mathrm{S}_{p} \mathrm{PCA}$ achieves the best result among all other methods for all dictionary sizes. The maximal recognition rates of $\mathrm{S}_{p} \mathrm{PCA}, \mathrm{RPCA}-\mathrm{OM}, \mathrm{SSPCA}, \mathrm{NMF}, \mathrm{ICA}$ and PCA are $97.20 \%, 87.82 \%, 96.35 \%, 93.32 \%, 91.46 \%$ and $91.08 \%$, respectively.

\subsubsection{MutliPIE database}

The CMU Multi-PIE [24] contains 337 subjects across simultaneous variation in pose, expression and illumination. We manually cropped the face portion of the image and then normalized it to $100 \times 80$. Analogous to the experimental settings in [37], all the 249 subjects among these 337 subjects in Session 1 were used as training set. To verify the robustness of the proposed approach to expression, four subsets with expression variations in Sessions 1, 2 and 3 were used for testing. For the training set, we used 3 frontal images of each subject (249 subjects in all) in session 1 with extreme illuminations $\{0,1,7\}$ and neutral expression (refer to Fig.6(a) for examples). For the 


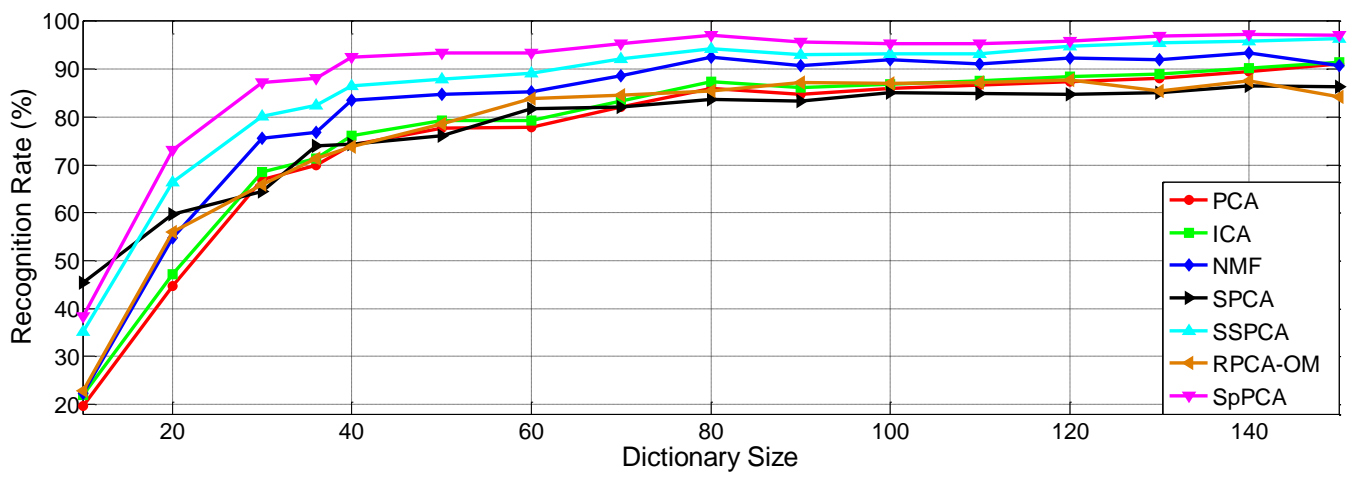

Figure 5: Classification accuracy versus dictionary size on Extended YaleB database

testing set, 4 typical frontal images with illuminations $\{0,2,713\}$ and different expressions (smile in Sessions 1 and 3, squint and surprise in Session 2) were used (refer to Fig. 6(b) for examples with surprise in Session 2, Fig. 6(c) for examples with squint in Session 2, Fig. 6(d) for examples with smile in Session 1, and Fig. 6(e) for examples with smile in Session 3). Table 2 lists the recognition rates in four testing sets by the competing methods (for $K=36$ ). From Table 2, we can see that the proposed method achieves the best performance on three testing sets, except Session 2 with squint expression. Especially, our method has more than $2 \%$ improvement over the second best method on Session 2 with surprise expression and Session 3 with smile expression. Meanwhile, the results in Table 2 also imply that face recognition with surprise expression change is much more difficult than face recognition with the other two expression changes.

Table 2: Face recognition rates (\%) on Multi-PIE database with $K=36$

\begin{tabular}{lccccccc}
\hline & PCA & ICA & NMF & SPCA & SSPCA & RPCA-OM & S $_{p}$ PCA \\
\hline Session1_smile & 65.96 & 64.56 & 60.24 & 55.72 & 62.15 & 66.26 & $\mathbf{6 6 . 9 7}$ \\
\hline Session2_squint & 35.54 & 36.14 & 28.92 & 19.13 & 34.94 & $\mathbf{3 6 . 4 4}$ & 35.09 \\
\hline Session2_surprise & 23.04 & 23.04 & 17.62 & 13.10 & 20.33 & 23.64 & $\mathbf{2 5 . 1 5}$ \\
\hline Session3_smile & 32.66 & 33.59 & 26.88 & 19.84 & 31.41 & 33.28 & $\mathbf{3 6 . 0 9}$ \\
\hline
\end{tabular}




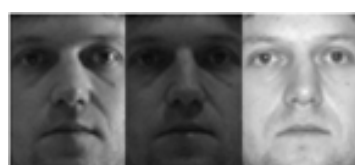

(a)

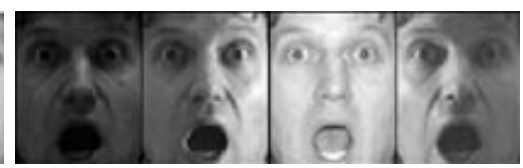

(b)

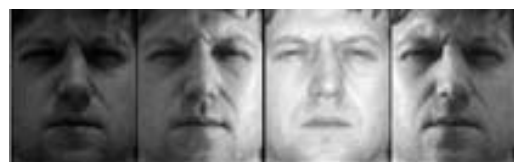

(c)

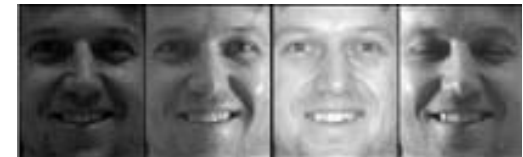

(d)

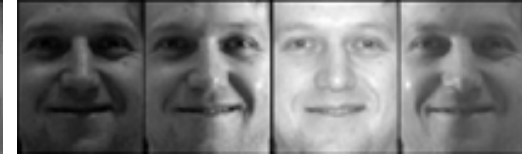

(e)

Figure 6: A subject in Multi-PIE database. (a) Training samples with only illumination variations. (b) Testing samples with surprise expression and illumination variations. (c) Testing samples with squint expression and illumination variations. (d) and (e) show the testing samples with smile expression and illumination variations in Session 1 and Session 3, respectively.

\section{Conclusions}

In this paper, we proposed to use low rank regularization to model the image-level structural dictionary elements. We introduced Schatten $p$-norm and an efficient optimization procedure to solve the proposed model. We evaluated the proposed method on denoising task of synthetic signals and face classification on AR, Extended YaleB and Multi-PIE databases with various variations, including occlusion, illumination and expression. The extensive experimental results demonstrated that $\mathrm{S}_{p} \mathrm{PCA}$ is more robust and effective than previous methods, such as SSPCA and SPCA.

\section{Acknowledgments}

The authors would like to thank the editor and the anonymous reviewers for their critical and constructive comments and suggestions. This work was partially supported by the National Science Fund for Distinguished Young Scholars under Grant Nos. 61125305, 914202016147218761233011 and 61373063, the Key Project of Chinese Ministry of Education under Grant No. 313030, the 973 Program No. 2014CB349303, Fundamental Research Funds for the Central Universities No. 30920140121005, and Program for Changjiang Scholars and Innovative Research Team in University No. IRT13072, National Natural Science Foundation for Young Scientists 
of China (Grant no. 61402289), National Science Foundation of Guangdong Province (Grant no. 2014A030313558), and Shenzhen Scientific Research and Development Funding Program (Grant no. JCYJ20140509172609171).

\section{References}

[1] I. Jolliffe, Principal component analysis, New York: Springer Verlag.

[2] P. Comon, Independent component analysis-a new concept?, Signal Processing 36 (1994) 287-314.

[3] C.-J. Lin, Projected gradient methods for non-negative matrix factorization, Neural Computation 19 (2007) 2756-2779.

[4] H.Zou, T.Hastie, R.Tibshirani, Sparse principal component analysis, J. Comput. Graph. Stat. 15 (2) (2006) 265-286.

[5] C. Croux, P. Filzmoser, H. Fritz, Robust sparse principal component analysis, Technometrics 55 (2) (2013) 202-214.

[6] L.Jacob, G.Obozinski, J.P.Vert, Group lasso with overlap and graph lasso, in: ICML, 2009.

[7] J.Huang, T.Zhang, D.Metaxas, Learning with structured sparsity, in: ICML, 2009.

[8] M. Yang, P. Zhu, F. Liu, L. Shen, Joint representation and pattern learning for robust face recognition, Neurocomputing (2015) 70-80.

[9] N. Shahid, N. Perraudin, V. Kalofolias, P. Vandergheynst, Fast robust pca on graphs, in: arXiv:1507.08173v1, 2015.

[10] R.Jenatton, G.Obozinski, F.Bach, Structured sparse principal component analysis, in: AISTATS, 2010.

[11] S. Ren, X. Qian, Structured sparse pca to identify mirna co-regulatory modules, in: ICASSP, 2014.

[12] Z. Szabo, B. Poczos, A. Lorincz, Online group-structured dictionary learning, in: CVPR, 2011. 
[13] E. J. Candes, X. Li, Y. Ma, J. Wright, Robust principal component analysis?, Journal of the ACM 58 (1) (2010) 1-73.

[14] G. Liu, Z. Lin, Y. Ma, Robust recovery of subspace structures by lowrank representation, IEEE Transactions on Pattern Analysis and Machine Intelligence 35 (1) (2013) 171-184.

[15] F. Zhang, J. Yang, Y. Tai, J. Tang, Double nuclear norm-based matrix decomposition for occluded image recovery and background modeling, IEEE Trans on Image Processing 24 (6) (2015) 1956-1966.

[16] J. Yang, J. Qian, L. Luo, F. Zhang, Y. Gao, Nuclear norm based matrix regression with applications to face recognition with occlusion and illumination changes, in: arXiv:1405.1207v1, 2014.

[17] L. Luo, J. Yang, J. Qian, Y. Tai, Nulcear-l1 norm joint regression for face reconstruction and recognition with mixed noise, Pattern Recognition 48 (2015) 3811-3824.

[18] R.G.Baraniuk, Compressive sensing, IEEE Signal Process 24 (4) (2007) 118-124.

[19] G.Marjanovic, V.Solo, On $l_{q}$ optimization and matrix completion, IEEE Trans. Signal Process 60 (11) (2012) 5714-5724.

[20] M. Malek-Mohammadi, M. Babaie-Zadeh, M. Skoglund, Performance guarantees for schatten-p quasi-norm minimization in recovery of lowrank matrices, Signal Processing 114 (2015) 225-230.

[21] F. Nie, H. Wang, H. Huang, C. Ding, Joint schatten $p$-norm and $l_{p^{-}}$ norm robust matrix completion for missing value recovery, Knowledge and Information Systems 42 (3) (2015) 525-544.

[22] A. Martinez, R. Benavente, The ar face database, Tech. rep., CVC Tech. Report (1998).

[23] K. Lee, J. Ho, D. Kriegman, Acquiring linear subspaces for face recognition under variable lighting, IEEE Transactions on Pattern Analysis and Machine Intelligence 27 (5) (2005) 684-698.

[24] R. Gross, I. Matthews, J. Cohn, T. Kanade, S. Baker, Multi-pie, Image and Vision Computing 28 (2010) 807-813. 
[25] M. Aharon, M. Elad, A. Bruchstein, K-svd:an algorithm for designing overcomplete dictionaries for sparse representation, IEEE Transactions on Signal Processing 54 (11) (2006) 4311-4322.

[26] M. Yang, L. Zhang, X. Feng, D. Zhang, Sparse representation based fisher discrimination dictionary learning for image classification, Int J Comput Vis 109 (2014) 209-232.

[27] H. Zheng, D. Tao, Discriminative dictionary learning via fisher discriminative k-svd algorithm, Neurocomputing (2015) 9-15.

[28] H. Nguyen, W. Yang, B. Sheng, C. Sun, Discriminative low-rank dictionary learning for face recognition, Neurocomputing (2016) 541-551.

[29] D.M.Witten, R.Tibshirani, T.Hastie, A penalized matrix decomposition, with applications to sparse principal components and canonical correlation analysis, Biostatistics 10 (3) (2009) 515.

[30] B. Recht, M. Fazel, P. A. Parrilo, Guaranteed minimum-rank solutions of linear matrix equations via nuclear norm minimization, SIAM Review 52 (3) (2010) 471-501.

[31] S. Boyd, N. Parikh, E. Chu, B. Peleato, J. Eckstein, Distributed optimization and statistical learning via the alternating direction method of multipliers, Foundation and Trends in Machine Learning 3 (1) (2011) $1-122$.

[32] J. Mairal, F. Bach, J. Ponce, G. Sapiro, Online dictionary learning for sparse coding, in: ICML, 2009.

[33] W. Cao, J. Sun, Z. Xu, Fast image deconvolution using closed-form thresholding formulas of $l_{q}$ regularization, Journal of Visual Communication and Image Representation 381 (13) (2013) 31-41.

[34] Y. Wang, W. Yin, J. Zeng, Global convergence of admm in nonconvex nonsmooth optimization, in: arXiv:1511.06324v2, 2015.

[35] X. Zhang, C. Xu, X. Sun, G. Baciu, Schatten-q regularizer constrained low rank subspace clustering model, Neurocomputing (2016) 36-47.

[36] F. Nie, J. Yuan, H. Huang, Optimal mean robust principal component analysis, in: ICML, 2012. 
[37] M. Yang, L. Zhang, J. Yang, D. Zhang, Robust sparse coding for face recognition, in: CVPR, 2011. 


\section{Algorithm 1 Solving Problem (4) by Inexact ALM}

1: Input: data matrix $\mathbf{X}$, dictionary size $K$, parameter $\lambda>0, \mu>0$

2: Initialize: dictionary $\mathbf{D}, \mathbf{V}=\mathbf{D}, \mathbf{Y}=0, \mathbf{Z}=0$

3: while not converge do

4: $\quad$ fix the others and update $\mathbf{Z}$ by

$$
\mathbf{Z}=\underset{\mathbf{Z}}{\arg \min }\|\mathbf{X}-\mathbf{D Z}\|_{F}^{2}, \quad \text { s.t. }\left\|\mathbf{Z}^{i}\right\|_{2} \leq 1 \forall i \in\{1,2, \cdots, K\}
$$

5: $\quad$ fix the others and update $\mathbf{D}$ by

$$
\mathbf{D}=\left(\mathbf{X Z}^{\mathbf{T}}+\frac{\mu \mathbf{V}-\mathbf{Y}}{2}\right)\left(\mathbf{Z Z}^{T}+\frac{\mu}{2} \mathbf{I}\right)^{-1}
$$

6: $\quad$ fix the others and update $\mathbf{V}$ by

$$
\begin{aligned}
\mathbf{V} & =\underset{\mathbf{V}}{\arg \min } \lambda \sum_{i=1}^{K}\left\|\Psi\left(\mathbf{V}_{i}\right)\right\|_{S_{p}}^{p}+\frac{\mu}{2}\left\|\mathbf{D}-\mathbf{V}+\frac{\mathbf{Y}}{\mu}\right\|_{F}^{2} \\
& =\underset{\mathbf{V}}{\arg \min } \lambda \sum_{i=1}^{K}\left(\left\|\Psi\left(\mathbf{V}_{i}\right)\right\|_{S_{p}}^{p}+\frac{\mu}{2 \lambda}\left\|\mathbf{V}_{\mathbf{i}}-\left(\mathbf{D}_{\mathbf{i}}+\frac{\mathbf{Y}_{\mathbf{i}}}{\mu}\right)\right\|_{F}^{2}\right) \\
& =\underset{\mathbf{V}}{\arg \min } \lambda \sum_{i=1}^{K}\left(\left\|\Psi\left(\mathbf{V}_{i}\right)\right\|_{S_{p}}^{p}+\frac{\mu}{2 \lambda}\left\|\Psi\left(\mathbf{V}_{\mathbf{i}}\right)-\Psi\left(\left(\mathbf{D}_{\mathbf{i}}+\frac{\mathbf{Y}_{\mathbf{i}}}{\mu}\right)\right)\right\|_{F}^{2}\right)
\end{aligned}
$$

7: $\quad$ update the multiplier

$$
\mathbf{Y}=\mathbf{Y}+\mu(\mathbf{D}-\mathbf{V})
$$

8: $\quad$ check the convergence condition

$$
\|\mathbf{D}-\mathbf{V}\|_{\infty} \leq \epsilon
$$

9: end while

10: Return dictionary $\mathbf{D}$ and coefficient $\mathbf{Z}$. 